\title{
Kawasaki disease with bronchopneumonia: A case report
}

\author{
*Dayana Nicholas ${ }^{1}$, Kenneth Nelson' ${ }^{1}$, Azmi Sarriff² \\ ${ }^{1}$ Clinical Pharmacy and Pharmacy Practice Unit, Faculty of Pharmacy, AIMST University, Kedah, Malaysia \\ ${ }^{2}$ Discipline of Clinical Pharmacy, School of pharmacy, University Sains Malaysia, Penang, Malaysia
}

\begin{abstract}
Kawasaki syndrome (KS), also known as Kawasaki disease (KD) is an acute systemic vasculitis that predominantly affects pre-school aged children. It is characterized by fever, bilateral nonexudative conjunctivitis, erythema of the lips and oral mucosa, changes in the extremities, rash, and cervical lymphadenopathy, and irritation and inflammation of the mouth, lips, and throat. Serious complications of KS include coronary artery dilatations and aneurysms, and KS is a leading cause of acquired heart disease. It has a predilection to coronary arteries, and its precise etiology is still unknown. Many infectious agents, including viruses and bacteria, have been suggested as potential causes of the disease. We presented the therapeutic management and clinical outcome of a 3 years old male patient, with a height of $96 \mathrm{~cm}$ and weight of 14.8 kilogram (underweight), who was referred to our hospital. Patient's symptoms and laboratory data confirmed Kawasaki disease accompanied with bronchopneumonia and treated along with antibiotics, aspirin and IV Immunoglobulin G (IgG), to maintain adequate antibodies levels, prevent infections and confer a passive immunity. The patient was successfully treated by administering IgG and antibiotics without any complications. Eventually, accurate diagnosis, more targeted treatment, and preventative strategies depend on the unraveling of the immunopathogenesis of this disease.
\end{abstract}

Key Words: Systemic vasculitis, children, coronary arteries, outcome, immunoglobulin G, antibodies.

\section{INTRODUCTION}

Kawasaki syndrome (KS), also known as Kawasaki disease (KD), is an acute febrile illness of unknown etiology that primarily affects children younger than 5 years of age. KS was first described in Japan by Tomisaku Kawasaki in 1967, and the first cases outside of Japan were reported in Hawaii in 1976 (Thomas, 2011).

Kawasaki disease is characterized by fever, bilateral nonexudative conjunctivitis, erythema of the lips and oral mucosa, changes in the extremities, rash, and cervical lymphadenopathy, and irritation and inflammation of the mouth, lips, and throat. Serious complications of KS include coronary artery dilatations and aneurysms, and KS is a leading cause of acquired heart disease in the United States.

\footnotetext{
*Corresponding Author:

Ms. Dayana Nicholas, Lecturer

Clinical Pharmacy and Pharmacy Practice Unit

Faculty of Pharmacy, AIMST University

Semeling, 08100 Bedong, Kedah, Malaysia

E-mail: dayanicholas@gmail.com

Contact No.: +60149476801
}

The standard treatment with intravenous immunoglobulin and aspirin substantially decreases the development of these coronary artery abnormalities. Seven cases of KD was reported who were admitted to Hospital University Sains Malaysia over a period of 8 years. Five children had all six criteria for diagnosis. Ileus was present in three and hydrops of the gall bladder and pneumonia in two each, but coronary artery dilatation was seen in only one patient. The epidemiological and clinical features of Malaysians with KD differ from those seen in Singapore and Japan, but are similar to those seen in Thailand, Australia and North America. The incidence of cardiac complications is low in Malaysians (Malik, 1996). Coronary artery aneurysms or ectasia develop in $\sim 15 \%$ to $25 \%$ of untreated children and may lead to ischemic heart disease or sudden death (Newburger et al., 2004).

We present this case where patient with KD who was successfully treated with $\operatorname{IgG}$ and antibiotics without any complications. 


\section{CASE REPORT}

Patient was a 3 years old male with a height of $96 \mathrm{~cm}$ and weight of 14.8 kilogram, who was underweight, admitted and referred by private clinic for having fever, cough and runny nose for 7 days. Patient has started having fever since 6 days ago (24 October 2011), a high grade fever of 38.5 degree Celsius $\left({ }^{\circ} \mathrm{C}\right)$. Patient also had feelings of chills and rigors; however there are no rashes, no bleeding tendency, and no specific pattern neither recent travelling outside/swimming in river. Patient refused to take oral medication, so he was given suppository paracetamol as a replacement of syrup paracetamol. Patient had febrile fit incident, patient suffered with generalized tonic-clonic seizure involving both the upper and lower limb and up rolling of eyes ball with clenching of teeth. However, there was no tongue bitting, urine/bowel incontinence nor cyanosis.

Patient was currently having fever associated with intermittent cheesy cough. Patient's eldest sister was previously having similar problem of, but she has already resolved. Patient was never admitted to the hospital and no surgery was done onto him before. Family has strong history of febrile fit but no epilepsy. Taken care by mother who was a housewife while father was an ex-army. Patient is the youngest out of 5 siblings. Other siblings also had febrile fit previously, currently all are healthy.

Patient was underweight with the weight of only 14.8 kilogram at the height of $96 \mathrm{~cm}$. However, patient was healthy, pink and alert, not tachypnoiec, temperature of the patient was spiking with $38.8^{\circ} \mathrm{C}$, and patient is mildly dehydrated. Respiratory rate was 30 and the pulse rate was 128 . There was some rashes over the patient's body, and patients tongue was strawberry red in colour with red fissured lips, muscular rashes noted over the face and trunk. This indicates the diagnosis for Kawasaki disease.

Fever spiking with high temperature and rashes all over the body indicates that the patient was suffering from Kawasaki's Disease. Also, patient was having cough intermittently due to suffering from bronchopneumonia.

To treat patient's spiking high fever, patient was administered with suppository paracetamol because patient was not tolerating orally. Intravenous penicillin was also given because patient has cough infections. Patient has low immune level hence he's being given intravenous immunoglobulin to maintain adequate antibodies levels to prevent infections and confers a passive immunity of the patient. Patient was given aspirin to reduce fever, and as anti-inflammatory medication.

\section{Laboratory Investigations}

Patient was diagnosed with Kawasaki's disease along with bronchopnuemonia and seizure. Laboratory investigations are shown in the Table 1. Temperature Monitoring $1^{\text {st }}$ day three times was $38.8^{\circ} \mathrm{C}, 38.8^{\circ} \mathrm{C}$ and $38.8^{\circ} \mathrm{C}$; for the second day was $37.0^{\circ} \mathrm{C}, 37.4^{\circ} \mathrm{C}$ and $37.4^{\circ} \mathrm{C}$

\section{DISCUSSION}

Definitive Kawasaki's Disease was confirmed after tracing the fever spiking with high temperature and rashes all over the body with strawberry fissured tongue. Also, patient was having cough intermittently due to suffering from bronchopneumonia.

$1^{\text {st }}$ day IV doses of Immunoglobulin G ( $\left.\operatorname{Ig} G\right)$ was started. After tracing all the blood investigations, IV drip1/2 SD5\%26cc/hourly, suppository paracetamol $125 \mathrm{mg} 6$ hourly was given since the patient was not tolerating oral administration. Till evening patient was having the same $38.8^{\circ} \mathrm{C}$ fever, suppository

Table 1: Laboratory investigations of Kawasaki's disease patient along with bronshopnuemonia and seizure.

\begin{tabular}{llcc}
\hline $\begin{array}{l}\text { Lab investiga- } \\
\text { tions }\end{array}$ & $\begin{array}{l}\text { Normal range } \\
\text { Pediatric value }\end{array}$ & $\mathbf{1}^{\text {st }}$ day & $2^{\text {nd }}$ day \\
\hline Urea & $2.5-5.0$ & - & $12.1^{*}$ \\
$\mathrm{Na}$ & $136-145$ & - & 136 \\
$\mathrm{~K}$ & $3.5-5.1$ & - & 3.4 \\
$\mathrm{Mg}$ & $0.6-1.0$ & - & 1.04 \\
$\mathrm{Ca}$ & $2.13-2.60$ & - & - \\
$\mathrm{Hb}$ & $11.5-16.5$ & 12.1 & - \\
$\mathrm{TWBC}$ & $4.1-10.9$ & $14.9^{*}$ & - \\
$\mathrm{Platelet}$ & $140-144$ & $404^{*}$ & - \\
$\mathrm{PCV}$ & $35-50$ & 37.4 & - \\
$\mathrm{L}$ & $20-40$ & 23.6 & - \\
$\mathrm{M}$ & $2.0-10$ & 7.1 & - \\
$\mathrm{G}$ & $40-80$ & 69.3 & - \\
$\mathrm{RBC}$ & $4.2-6.5$ & - & - \\
ESR & $3.0-13.0$ & - & $74^{*}$ \\
ASOT & & $<200$ & \\
\hline
\end{tabular}

*Values with significance 
paracetamol $125 \mathrm{mg}$ dose was increased to $250 \mathrm{mg}$ q.i.d (four times a day) and the patient was diagnosed with bronchopneumonia, hence patient was given IV C/ penicillin 370000 ii stat (given 6 hourly).

From the beginning of $2^{\text {nd }}$ day, IV IgG $30 \mathrm{gm}$ (600mls) over 10 hourly was started. IV C pencillin 370000 ii 6 hourly was continued and Syrup aspirin was also given $300 \mathrm{mg}$ q.i.d. Paracetamol suppository $250 \mathrm{mg}$ q.i.d was inserted.

$3^{\text {rd }}$ day IV Ig completed with no reaction but continued with other medications for the third day. The situation of the patient was progressed and the temperature was normal.

\section{Drug Related Issue and Precaution}

IV C Penicillin is an antibiotic which is used to treat bacterial infections. Patient was having cough and high grade fever, perhaps due to bacterial infection. Thus, penicillin intravenously was used because the patient does not want to take his medication orally. Suppository Paracetamol is the acetaminophen used as analgesics and antipyretics. Suppository was given to reduce the spiking temperature. IV Ig is an expensive therapy that was used for the patient who needs high doses of antibody for the acute infections. This Immunoglobulin acts as a plasma protein replacement therapy for immune deficient patients who have decreased or abolished antibody production capabilities. Therefore, IV Ig was administered to maintain adequate antibodies levels to prevent infections and confers a passive immunity.

\section{Medication Related Problem and Outcome}

Aspirin is used to relieve minor aches and pains, as an antipyretic to reduce fever, and as anti inflammatory medication. In children with Kawasaki's disease, aspirin is taken at dosages based on body weight, initially q.i.d for up to two weeks and then at a lower dose once daily for a further six to eight weeks. The dosage given was rather high since the patient is very small and underweight. Action to be taken is administration of aspirin in the patient must be reduced to lower dose to prevent aspirin poisoning. Aspirin is also considered to be a precaution treatment to prevent any complications such as coronary aneurysms. But in this case the patient was still in progress. Corticosteroids also have been used to treat patients who have failed to respond to initial therapy for Kawasaki disease (Shulman, 2003).

\section{Outcome and Follow-Up}

The patient was recovered completely. His last haemogram, few days after the initial admission showed complete resolution of the disease.

\section{CONCLUSION}

Although the cause of KD is currently unknown, it is apparently related to the interaction of genetic and environmental factors, with ongoing research focusing primarily on an infectious etiology rather than an unusual reaction to a therapeutic agent (Meissner and Leung, 2000).

The correct diagnosis can make out from the symptoms for Kawasaki's test. There is no diagnosis test or confirmatory test is available to detect or diagnose the Kawasaki disease so far. The success of early treatment and optimum therapy can be a lifesaving factor for Kawasaki disease in children and saving them from serious complications like coronary aneurysms. Role of corticosteroids also can highly respond to KD but these medications are associated with a long list of potential adverse reactions including thrombosis, hypertension.

Some antibiotics and corticosteroids may decrease anti-streptococcal antibody titres (ASOT) levels. Here the patient was slowly recovered taking IgG and antibiotics with aspirin. Ultimately, accurate diagnosis, more targeted treatment, and preventative strategies depend on the unraveling of the immunopathogenesis of this disease.

\section{REFERENCES}

Malik AS (1996) Kawasaki disease in Malaysia. Annals of Tropical Paediatrics, 16(3): 213-220. PMid:8893951

Meissner HC, Leung DY (2000) Superantigens, conventional antigens and the etiology of Kawasaki syndrome. Pediatric Infectious Disease Journal, 19(2): 91-94. [DOI] PMid:10693992

Newburger JW et al. (2004) Diagnosis, treatment and longterm management of Kawasaki disease, Journal of the American Heart Association, 110(17): 2747-2771. [DOI]

Shulman ST (2003) Is there a role for corticosteroids in Kawasaki disease?, The Journal of Pediatrics, 142(6): 601603. [DOI] PMid:12838185

Thomas RF (2011). Kawasaki syndrome. URL 